\title{
Manifestaciones clínicas del dengue hemorrágico en Puerto Rico, 1990-19911
}

\author{
José G. Rigau-Pérez ${ }^{2}$ y Asociación de Epidemiólogos de Puerto Rico ${ }^{3}$
}

RESUMEN Este estudio se realizó con el fin de mostrar que el dengue hemorrágico (DH) se da en Puerto Rico, si bien es una enfermedad subnotificada, lo que se debe en parte a su subdiagnóstico en los hospitales. La vigilancia de casos graves de dengue permitió determinar que hubo 986 hospitalizaciones por sospecha de dengue en el período de 1990-1991. En esa época, el sistema de vigilancia identificó 20 casos de DH, incluidos tres de síndrome de choque por dengue (SCD). Nuestra subsecuente revisión de los expedientes de los 986 pacientes reveló que en 102 de ellos había razones para apoyar el diagnóstico clínico de DH (88) y de SCD (14). Entre estos 102 había 57 que dieron resultados virológicos o serológicos positivos a dengue y que reunían los criterios de caso de dengue establecidos por la OMS (fiebre, manifestaciones hemorrágicas, trombocitopenia y excesiva permeabilidad capilar). En el grupo de 57 pacientes, la media de edad era de 38 años y preponderaban los hombres (34, o sea 59,3\%), incluidos ocho casos de SCD y dos (3,5\%) defunciones (en mujeres de 16 y 55 años de edad). Los sintomas hemorrágicos fueron leves y con frecuencia se observaron hemoconcentración, hipoalbuminemia y concentraciones elevadas de aspartato y alanina aminotransferasas. Las hospitalizaciones duraron unos 5 días en término medio. La descripción clínica de estos casos de DH en Puerto Rico coincide con descripciones anteriores de la enfermedad en la literatura médica, pero la distribución por edad de los pacientes es similar al patrón que se suele encontrar en las Américas (donde afecta a todos los grupos de edad), en contraste con el de Asia Sudoriental (donde afecta principalmente a niños pequeños). El número de casos identificados en nuestro estudio fue casi tres veces mayor que el número notificado por el sistema de vigilancia establecido. Nuestros resultados indican que es necesario mejorar la capacidad de los clinicos locales para reconocer y notificar los casos de DH.

El dengue es una enfermedad aguda transmitida por mosquitos y causada por cuatro serotipos de virus (DEN-1,

\footnotetext{
Se publicó en inglés en la Revista Panamericana de Salud Pública/Pan American Journal of Public Health, Vol. 1, No. 5, 1997, con el título "Clinical manifestations of dengue hemorrhagic fever in Puerto Rico, 1990-1991".

2 Centros para el Control y la Prevención de Enfermedades, Centro Nacional de Enfermedades Infecciosas, San Juan, Puerto Rico. Dirección postal: San Juan Laboratories, CDC, 2 calle Casia, San Juan, Puerto Rico 00921-3200.

3 La Asociación de Epidemiólogos de Puerto Rico es una organización voluntaria multidisciplinaria de profesionales dedicados al ejercicio de la epidemiología y del control de infecciones en Puerto Rico.
}

DEN-2, DEN-3 o DEN-4), que se caracteriza por fiebre, cefalea, mialgia $y$ erupción cutánea de comienzo repentino. La forma endémica, que muestra una creciente incidencia en la mayor parte de las zonas tropicales, es una de las enfermedades microbianas emergentes que amenazan al mundo $(1,2)$. Recientemente han ocurrido extensos brotes de dengue y dengue hemorrágico $(\mathrm{DH})$ en Centroamérica y América del Sur, así como en las islas del Caribe. La transmisión del dengue en esos territorios se ha atribuido a un único vector, el mosquito Aedes aegypti.
La fiebre del dengue (FD), que suele ser autolimitante, no amenaza la vida. El DH, potencialmente mortal, es más común en Asia Sudoriental, donde se han notificado más de un millón de casos de 1986 a 1990 y donde la enfermedad es causa frecuente de hospitalización y muerte en niños (3). La característica que distingue al DH de la FD no es la aparición de hemorragia, sino más bien el escape de plasma por mayor permeabilidad capilar, que puede llevar de forma insidiosa $y$ rápida al síndrome de choque por dengue (SCD) con hipotensión o choque 
manifiesto (4-7). Aunque el choque del dengue puede revertirse con una administración agresiva de liquidos, la clave del éxito terapéutico está en su prevención. Después de manifestado el choque, la tasa de letalidad puede ser superior a $10 \%$, aun en centros especializados (7-10). Además, hay casos graves y mortales que no se presentan con escape de plasma sino con hemorragia gastrointestinal grave desde el principio (11).

El primer caso de DH en las Américas se confirmó en Puerto Rico en 1975 (12). A pesar de una vigilancia activa y de la aparición de una epidemia de DH en Cuba en 1981 y de los brotes de DH que ocurrieron en México (1984) y Nicaragua (1985), solo se habían documentado tres casos en Puerto Rico para fines de 1985 (13-15). No obstante, al año siguiente una epidemia se extendió por toda la isla y causó 29 casos de DH (incluidas tres defunciones), cuya etiología se estableció por métodos serológicos o virológicos (15, 16). Si bien desde entonces y para 1994 se notificaron varios brotes de $\mathrm{DH}$ en Centroamérica y América del Sur, solo un pequeño número de casos anuales fue registrado en Puerto Rico $(17,18)$.

El presente estudio se emprendió para demostrar que el DH, según fuera definido por la OMS (19) y descrito en el Asia Sudoriental, está presente en Puerto Rico pero que la enfermedad está subnotificada, debido en parte a su subdiagnóstico en los hospitales. Al respecto, se discute la repercusión de nuestros resultados en el diagnóstico y tratamiento hospitalarios del DH.

\section{MATERIALES Y MÉTODOS}

\section{Definiciones de casos clínicos}

La OMS ha establecido cuatro criterios para notificar el DH: 1) fiebre; 2) manifestaciones hemorrágicas, que incluyen por lo menos una prueba del torniquete positiva (excepto en casos de choque) y más tarde fenómenos hemorrágicos leves o graves; 3) trombocitopenia (100 000/ $\mathrm{mm}^{3}$ ó menos); 4) hemoconcentración (aumento del hematocrito de 20\% o más), o bien pruebas objetivas de permeabilidad capilar (19). Dado que una prueba del torniquete positiva es en sí un fenómeno hemorrágico provocado de carácter leve, para los fines de este estudio se interpretó el segundo criterio como cualquier manifestación hemorrágica, incluso fenómenos hemorrágicos menores tales como los implicados en una prueba del torniquete positiva. Siguiendo las pautas de la OMS, se estimó que la hipoalbuminemia o las efusiones pleurales o abdominales (documentadas por radiografía, ultrasonografía o tomografía computadorizada), o ambas afecciones, eran pruebas objetivas de la permeabilidad capilar citada en el cuarto criterio.

El síndrome de choque del dengue se definió de acuerdo con los criterios antedichos, sumados a un descenso de la tensión del pulso ( $\leq 20 \mathrm{~mm} \mathrm{Hg}$ ). Para determinar la gravedad del DH se recurrió a los grados establecidos por la OMS (19): I) una prueba del torniquete positiva como única manifestación hemorrágica; II) hemorragia espontánea; III) signos de insuficiencia circulatoria (descenso de la tensión del pulso o hipotensión); IV) choque profundo con tensión arterial y pulso indetectables. Los casos de grados III y IV corresponden a casos de SCD.

\section{Detección de posibles casos de DH}

La Sección de Dengue de los Centros para el Control y la Prevención de Enfermedades (CDC) de los Estados Unidos recibe muestras de sangre de presuntos enfermos de dengue o DH, remitidas por instituciones públicas y privadas de Puerto Rico (20-21). La información demográfica y clínica básica y un registro de los resultados de las pruebas diagnósticas de laboratorio se incorporan a una base de datos sistematizada en computadora.

Un sistema de vigilancia paralelo, independiente del vinculado a la recolección de muestras con fines diagnósticos, se ha hecho posible por medio de los informes de profesionales vinculados con el control de infecciones en los hospitales, quienes están organizados como Asociación de Epidemiólogos de Puerto Rico. La entidad provee información clínica acerca de los pacientes internados y los divide en casos de dengue sospechados o verdaderos. El formulario de notificación empleado por esta entidad incluye alrededor de 40 rubros relacionados con signos, síntomas y resultados de laboratorio correspondientes a $\mathrm{DH}$.

En 1991, los informes del Instituto de Medicina Forense y del Registro Demográfico de Puerto Rico fueron también incluidos en la recopilación. Para el análisis de los informes de 1990 y 1991 recibidos de las cuatro fuentes mencionadas (los CDC, los profesionales dedicados al control de infecciones, el Instituto de Medicina Forense y el Registro Demográfico), se empleó un algoritmo de detección en computadora, lo que permitió la identificación de las defunciones, de los pacientes que cumplimentaban los criterios de DH y SCD establecidos por la OMS, y también de los que respondían a solo tres de los cuatro criterios. Para esa etapa del tamizaje se definió la hipoalbuminemia como una concentración de seroalbúmina menor de $3 \mathrm{~g} / \mathrm{dL}$. Los casos identificados por medio del algoritmo habían sido seleccionados mediante una revisión de registros hospitalarios llevada a cabo por uno de los autores.

\section{Análisis de los registros hospitalarios}

Para identificar las pruebas con resultados anormales en el registro hospitalario de un paciente, recurrimos a los estándares de cada hospital en relación con seroalbúmina, proteína, aspartato-aminotransferasa (ASAT, antes transaminasa glutamicooxalacética o GOT sérica) y alanina-aminotransferasa (ALAT, antes transaminasa glutamicopirúvica o GPT sérica), tiempo de protrombina y tiempo parcial de tromboplastina. El valor normal asignado para bilirrubina total fue $\leq 1 \mathrm{mg} / \mathrm{dL}$ (22-23). Como la fiebre puede aumentar el número de eritrocitos observados en el sedimento urina- 
rio, la microhematuria se definió por la presencia de cinco eritrocitos o más por campo de alta resolución o bien por positividad a la sangre en la prueba de orina (24). La abstracción de los datos se centró en los criterios diagnósticos de $\mathrm{DH}$, dejando de lado la descripción de todos los hallazgos clínicos. Los casos se agruparon según el mes en que comenzaron los síntomas. En razón del pequeño tamaño de los grupos y de la falta de aleatoriedad de las muestras, no se realizaron pruebas de significación tendientes a demostrar diferencias entre los grupos.

\section{Métodos de laboratorio}

En relación con las pruebas diagnósticas efectuadas en el transcurso de la enfermedad, las muestras de suero obtenidas menos de 6 días después del comienzo de la afección fueron procesadas para el aislamiento del virus mediante el cultivo de células de mosquito C6/36 o por inoculación de mosquitos Toxorhynchites (25-27). Los virus del dengue se identificaron mediante el uso de anticuerpos monoclonales específicos contra cada serotipo en una prueba de inmunofluorescencia indirecta con cultivos de células infectadas por el virus o tejidos de mosquitos inoculados. Los especímenes de suero también fueron analizados por inmunoensayo enzimático de captación de anticuerpos IgM (MAC-ELISA) (28-30), con fines de detectar IgM antidengue. Las muestras de las que se había aislado el virus fueron evaluadas más tarde por la prueba de inhibición de la hemaglutinación (IH) o por IgGELISA, con objeto de determinar si la infección era primaria o secundaria, según fueran las concentraciones de anticuerpos antidengue en los sueros de la fase aguda y de convalecencia.

Además, los especímenes que dieron resultados dudosos por MACELISA fueron analizados posteriormente por IH o IgG-ELISA en un intento por confirmar el diagnóstico sobre la base de la detección de una respuesta anamnésica de anticuerpos antidengue (30-32).

\section{Definiciones de casos en el laboratorio}

La confirmación de una infección en curso por virus del dengue se basó en los siguientes criterios: 1) aislamiento del virus en muestras de suero o tejidos de autopsia, o 2) seroconversión de negativo a positivo o bien un aumento cuádruple o mayor de los títulos de anticuerpos antidengue en sueros pareados. Por inmunofluorescencia o inmunocitoquímica (33) se intentó demostrar la presencia de antígeno viral en tejidos de autopsia, pero en todos los casos los resultados fueron negativos.

Una infección primaria en curso fue definida como 1) positividad a IgM (densidad óptica $>0,20$ ) en una muestra de suero correspondiente a la fase aguda ( $\leq 5$ días después del comienzo de la enfermedad), a la vez negativa a anticuerpos antidengue por IH (título $<10)$, pero con una muestra de suero de la fase convaleciente ( $\geq 14$ días después del comienzo) con un título $<2560$ de anticuerpos antidengue por $\mathrm{IH}$; o 2) un caso en que se hubiera aislado virus de una sola muestra de suero tomada en la fase aguda, en ausencia de anticuerpos antidengue detectables por IH.

Una infección secundaria en curso se definió como 1) un caso con una muestra de suero tomada en la fase aguda que fuera positiva a IgM y a anticuerpos detectables por $\mathrm{IH}, \mathrm{y}$ con una muestra de suero tomada en la fase convaleciente con títulos de anticuerpos $\geq 2560$ en la IH, o 2) un caso en que el aislamiento de virus se hubiera logrado de una sola muestra de suero tomada en la fase aguda y en la que también se hubieran detectado anticuerpos antidengue por $\mathrm{IH}(34,35)$.

Se definieron como casos probables de dengue aquellos con 1) una concentración estable de anticuerpos antidengue, detectada por IH en sueros pareados, y con positividad a IgM en cualquiera de las dos muestras, o 2) con una muestra única de suero positiva a IgM, o con títulos de anticuerpos $\geq 1280$ en la IH, o títulos de anticuerpos IgG $\geq 163840$ en el ELISA. Estos casos se consideraron probables, y no verdaderos, porque los pacientes podrían haber sufrido dengue en los 3 meses anteriores - la IgM puede persistir 90 días o más-y los síntomas en el momento de recolectar las muestras de sangre podrían haber sido consecuencia de una enfermedad distinta del dengue $(28,29)$.

A menos que se indique lo contrario, los casos probables y confirmados se tomaron en su conjunto como casos diagnosticados por laboratorio o con positividad en las pruebas de laboratorio. Las muestras únicas que habían sido negativas a virus y a $\operatorname{IgM}$, y que además habían sido obtenidas más de 6 días después del comienzo de la enfermedad, no se consideraron diagnósticas y estos casos se consideraron indeterminados. En los especímenes recolectados 6 o más días tras el comienzo de los síntomas, la ausencia de IgM descartó el diagnóstico de dengue; por tanto, esos casos se consideraron negativos.

\section{RESULTADOS}

En 1990 y 1991, los profesionales dedicados al control de infecciones intrahospitalarias, el Instituto de Medicina Forense y el Registro Demográfico proporcionaron 986 informes de hospitalizaciones por presuntos casos de dengue en Puerto Rico. Esta cifra solo representa 5,7\% de los 17328 casos de dengue notificados a los CDC para ese período de 2 años. A partir de los signos y síntomas registrados en los 986 informes de hospitalización o defunción y de los resultados de las muestras diagnósticas remitidas al sistema de vigilancia basado en el laboratorio, se identificaron 17 casos de DH y tres de SCD con diagnóstico positivo por laboratorio. Esos 20 casos fueron los que el sistema pudo detectar rutinariamente, y son, por consiguiente, los que constituyeron el número de casos de DH notificados.

Mediante el análisis de los 966 informes restantes se identificaron 199 casos posibles de $\mathrm{DH}$; dos informes basados en consultas ambulatorias se 
eliminaron de estudios ulteriores. Posteriormente, se revisaron los registros hospitalarios de los 20 casos de DH y de los 197 casos posibles. Los 217 registros de hospitalización sustentaron un diagnóstico clínico de DH en 88 casos y de SCD en 14. Si bien los registros de los 115 casos restantes eran compatibles con un diagnóstico clínico de FD, muchos no se pudieron clasificar con mayor precisión por falta de información sobre los siguientes síntomas o pruebas: fiebre (2), prueba del torniquete (103), sangre en materia fecal (97), sangre en orina (4), repetición del hematocrito (5) y evaluación de seroalbúmina (20). Cabe señalar que este grupo incluyó dos de los 20 casos de DH que fueron notificados en un principio pero cuyos registros no mostraban pruebas suficientes para satisfacer los cuatro criterios diagnósticos de DH (no se mencionaba la fiebre en un caso ni la hemorragia en otro). La tasa de letalidad de los 115 pacientes fue de $5,2 \%$; es decir, que ocurrieron seis defunciones.

Cincuenta y tres $(46,1 \%)$ de los 115 casos de FD fueron positivos por laboratorio. De ellos, 15 (28,3\%) egresaron con diagnóstico de $\mathrm{DH}$, aunque en los registros de 11 no constaba el antecedente de permeabilidad capilar excesiva. En cambio, el dengue no había sido mencionado en los diagnósticos de defunción de los tres pacientes $(5,7 \%)$ que murieron en el subgrupo correspondiente a pacientes positivos a FD según pruebas de laboratorio. Esas defunciones ocurrieron en un hombre de 61 años con diagnóstico de perforación de úlcera pilórica, peritonitis y coma hepático; una mujer de 35 años con diagnóstico de leucemia mielomonocítica aguda y hepatitis química, además de hemorragia intracerebral y hepatitis activa crónica; y una mujer de 48 años con diagnóstico de insuficiencia hepática. En las historias clínicas de los tres casos fatales no se registraban antecedentes de enfermedad hepática.

Los 102 casos que según nuestra revisión eran clínicamente compatibles con DH o SCD incluyeron 57 con resultados positivos por métodos serológicos o virológicos, 29 con resultados de laboratorio indeterminados, y 16 con resultados negativos. De los 57 positivos, 42 mostraban resultados que sugerían FD; 11 fueron confirmados por métodos serológicos y 4 por aislamiento del virus, que fue DEN-1 en un caso, DEN-2 [genotipo del grupo II (36)] en 2, y DEN-4 en el restante.

De los 15 casos confirmados, uno podría caracterizarse como respuesta inmune primaria, en tanto que los otros 14 podrían considerarse infecciones secundarias. De los 16 con resultados negativos, solo dos recibieron diagnósticos que claramente apuntaban a otras afecciones (apendicitis supurada aguda en un caso y leucemia en el otro). El cuadro 1 muestra las características de los casos que se ciñeron a los criterios clínicos de DH, agrupados según los resultados de laboratorio en relación con el diagnóstico de dengue: positivo, indeterminado o negativo.

Los grupos con diagnóstico positivo e indeterminado tuvieron características similares, pero los pacientes con resultados negativos por laboratorio presentaron diferencias, puesto que en este grupo la proporción de los dos sexos fue igual, la media de edad fue mucho menor que en los otros dos grupos, y no habían ocurrido defunciones. En los pacientes con resultados indeterminados y negativos, la mediana de los meses en que comenzaron los síntomas correspondió a agosto, que es cuando habitualmente se da el aumento anual de la incidencia de dengue. En cambio, en los casos positivos según las pruebas de laboratorio, el mes correspondiente a la mediana fue octubre, época de máxima incidencia anual de dengue o cercana a ella. La media en cuanto a tiempo de hospitalización fue mayor (8,9 días) en los pacientes con resultados negativos.

Se trazó un perfil de la progresión de la enfermedad (cuadro 2) calculando la media del tiempo transcurrido desde el comienzo de los síntomas hasta el ingreso al hospital, el valor máximo de hematocrito, el valor mínimo de hematocrito, el máximo recuento de plaquetas y la tensión arterial diastólica más baja, así como el tiempo transcurrido hasta el egreso del hospital. El valor máximo de hematocrito (señal de hemoconcentra-

CUADRO 1. Características de los casos con diagnóstico clínico y confirmación de dengue hemorrágico por laboratorio. Puerto Rico, 1990-1991

\begin{tabular}{|c|c|c|c|}
\hline \multirow[b]{2}{*}{ Variable } & \multicolumn{3}{|c|}{ Diagnóstico de dengue por laboratorio } \\
\hline & $\begin{array}{l}\text { Positivo } \\
(n=57)\end{array}$ & $\begin{array}{l}\text { Indeterminado } \\
\quad(n=29)\end{array}$ & $\begin{array}{l}\text { Negativo } \\
(n=16)\end{array}$ \\
\hline Razón de hombres a mujeres & $1,5: 1$ & $2,2: 1$ & $1: 1$ \\
\hline $\begin{array}{l}\text { Edad media } \\
\text { Recorrido }\end{array}$ & $\begin{array}{l}38 \text { años } \\
4-86 \text { años }\end{array}$ & $\begin{array}{c}35 \\
7 \text { meses-91 años }\end{array}$ & $\begin{array}{c}27 \\
10-73 \text { años }\end{array}$ \\
\hline Defunciones & $\begin{array}{l}2(3,5 \%) \text { mujeres } \\
\text { de } 16 \text { y } 55 \text { años }\end{array}$ & $\begin{array}{l}3(10,3 \%) \text { hombres de } \\
34 \text { y } 39 \text { años y una } \\
\text { mujer de } 85\end{array}$ & 0 \\
\hline $\begin{array}{l}\text { Mes de comienzo de síntomas } \\
\text { (mediana) }\end{array}$ & Octubre & Agosto & Agosto \\
\hline Lugar de residencia $^{a}$ & 24 & 17 & 14 \\
\hline Hospitales $^{a}$ & 20 & 15 & 7 \\
\hline Atención en hospital privado & $39(68,4 \%)$ & $15(51,7 \%)$ & $11(68,8 \%)$ \\
\hline $\begin{array}{l}\text { Duración de la estancia } \\
\text { en el hospital (en días) }\end{array}$ & & & \\
\hline $\begin{array}{l}\text { mediana } \\
\text { recorrido } \\
\text { media }\end{array}$ & $\begin{array}{c}5 \\
2 \text { a } 42 \\
6,8\end{array}$ & $\begin{array}{c}5 \\
0 \text { a } 19 \\
6,0\end{array}$ & $\begin{array}{c}5 \\
2 \text { a } 39 \\
8,9\end{array}$ \\
\hline
\end{tabular}

a Puerto Rico tiene 78 municipalidades y 65 hospitales que pueden notificar casos. 
CUADRO 2. Perfil de la progresión de la enfermedad en 102 casos hospitalizados con diagnóstico clínico de dengue hemorrágico, según el diagnóstico de laboratorio. Puerto Rico, 1990-1991

\begin{tabular}{lccc}
\hline & \multicolumn{3}{c}{ Diagnóstico de dengue por laboratorio } \\
\cline { 2 - 4 } \multicolumn{1}{c}{ Variable } & $\begin{array}{c}\text { Positivo } \\
(n=57)\end{array}$ & $\begin{array}{c}\text { Indeterminado } \\
(n=29)\end{array}$ & $\begin{array}{c}\text { Negativo } \\
(n=16)\end{array}$ \\
\hline Media de días desde comienzo de los síntomas hasta: & & \\
Admisión & 4,7 & 3,8 & 5,6 \\
Hematocrito mayor & 5,8 & 4,2 & 7,4 \\
Hematocrito menor & 7,5 & 7,0 & 10,1 \\
Recuento más bajo de plaquetas & 6,6 & 5,4 & 6,1 \\
Tensión arterial diastólica menor & 6,6 & 5,4 & 6,5 \\
Egreso & 11,4 & 9,6 & 14,6 \\
\hline
\end{tabular}

ción) en los casos positivos se observó, en promedio, un día antes de que el recuento de plaquetas y la tensión arterial diastólica descendieran a niveles mínimos. Ese fue también el patrón mostrado por los casos que se consideraron indeterminados. En cambio, en los casos negativos, el valor máximo de hematocrito se alcanzó, en promedio, un día después de que el recuento de plaquetas y la tensión diastólica descendieran a valores mínimos.

En la mayoría de los 57 pacientes de $\mathrm{DH}$, así identificados por resultados de laboratorio positivos según la revisión de los registros hospitalarios (cuadro 1), se había citado al dengue en el diagnóstico de internación (35 casos, ó $61,4 \%$ ) o egreso ( $45,78,9 \%)$; el resto se internó o se dio de alta con diagnóstico de trombocitopenia, hemorragia o síndrome viral. A seis de los 57 pacientes $(10,5 \%)$ se les diagnosticó DH cuando fueron admitidos, pero solo cuatro continuaban siendo portadores del virus al egresar. A otros 19 se les había diagnosticado $\mathrm{DH}$ en el momento del egreso, de modo que $23(40,4 \%)$ de los 57 casos de DH con diagnóstico positivo por laboratorio habían recibido el diagnóstico correcto cuando egresaron. Diecisiete pacientes $(29,8 \%)$ provenían de un solo hospital localizado en la zona metropolitana de San Juan; ningún otro hospital notificó más de seis $(10,5 \%)$ de los 57 casos.

Los cuadros 3 y 4 muestran la frecuencia de los signos y síntomas, más algunos resultados de los análisis clíni- cos de laboratorio, de los 57 pacientes con DH o SCD que tuvieron resultados de laboratorio positivos. Conforme los requisitos de la OMS para la definición de un caso, todos presentaban fiebre, manifestaciones hemorrágicas, trombocitopenia y algunas pruebas de permeabilidad capilar anormal (efusio-

CUADRO 3. Signos y síntomas en 57 pacientes hospitalizados con dengue hemorrágico confirmado por laboratorio, Puerto Rico, 1990-1991

\begin{tabular}{lrc}
\hline & \multicolumn{2}{c}{ Pacientes $^{\mathrm{a}}$} \\
\cline { 2 - 3 } Síntomas o signos & No. & $(\%)$ \\
\hline Fiebre & 57 & $(100,0)$ \\
Erupción & 27 & $(47,4)$ \\
Hepatomegalia & 6 & $(10,5)$ \\
Derrame seroso & 3 & $(5,3)$ \\
Choque & 3 & $(5,3)$ \\
Tipos de hemorragia & 57 & $(100,0)^{\mathrm{b}}$ \\
Hematuria microscópica & 28 & $(51,9)^{\mathrm{c}}$ \\
Petequias & 26 & $(45,6)$ \\
Epistaxis & 13 & $(22,8)$ \\
Hemorragia gingival & 8 & $(14,0)$ \\
Sangre en materia fecal & 8 & $(14,0)$ \\
Prueba del torniquete positiva & 5 & $(31,3)^{\mathrm{d}}$ \\
Sangre en vómito & 4 & $(7,0)$ \\
Sangrado en sitio de & & \\
inyección o venopuntura & 4 & $(7,0)$ \\
Hemoptisis & 3 & $(5,3)$ \\
Hemorragia vaginal & 2 & $(3,5)$ \\
Hematuria macroscópica & 2 & $(3,5)$ \\
Otras hemorragias & 2 & $(3,5)$ \\
\hline
\end{tabular}

a Estimación mínima, puesto que la búsqueda no fue uniforme para todos los pacientes. No obstante, el denominador fue 57 salvo en los casos en que se indica lo contrario.

b Solo $2(3,5 \%)$ pacientes tuvieron manifestaciones hemorrágicas graves.

${ }^{c}$ Denominador $=54$

${ }^{\mathrm{d}}$ Denominador $=16$. nes, baja concentración de albúmina o hemoconcentración). Las manifestaciones hemorrágicas más frecuentes fueron hematuria microscópica y petequias; solo dos pacientes $(3,5 \%)$ sufrieron hemorragias graves o copiosas. $\mathrm{Si}$ bien las concentraciones de ASAT y ALAT a menudo estuvieron solo ligeramente elevadas, en $5(10,0 \%)$ y 7 $(14,6 \%)$ de los casos en que se determinaron sobrepasaron, respectivamente, las $500 \mathrm{U} / \mathrm{L}$. Las alteraciones del tiempo parcial de tromboplastina fueron más frecuentes y pronunciadas que las del tiempo de protrombina. La razón de las concentraciones de ASAT a las de ALAT varió de 8:100 a 3,96:1 y fue menor de 2:1 en $40(85,1 \%)$ de los 47 pacientes en que se evaluaron. Se observó un patrón similar en los casos de $\mathrm{DH}$ diagnosticados según criterios clínicos pero con resultados de laboratorio negativos o indeterminados.

No hubo pacientes en quienes una prueba positiva del torniquete fuera la única manifestación hemorrágica $(\mathrm{DH}$ grado I), pero sí hubo 49 casos de DH de grado II, 5 de grado III (SCD) y 3 de grado IV, entre ellos dos defunciones. De estas, una correspondió a una joven de 16 años con DH confirmado e infección secundaria del dengue, que fue internada en estado de coma con convulsiones tónico-clónicas. En el transcurso de las primeras 24 horas de internación presentó hipotensión y murió de paro cardiorrespiratorio a los 10 días de la hospitalización. La otra defunción ocurrió en una paciente de 55 años con DH confirmado e infección por el virus DEN-2 con respuesta secundaria. Esta paciente presentó fiebre, trombocitopenia (recuento de plaquetas: $27000 / \mathrm{mm}^{3}$ ) e hipotensión en las primeras 24 horas de la internación, y murió de paro cardiorrespiratorio a los 3 días de la hospitalización. En este segundo caso, los diagnósticos de defunción principales fueron insuficiencia hepática y pancreatitis; el dengue no figuraba entre ellos.

Otras enfermedades infecciosas cuya exploración mediante otras pruebas de laboratorio se efectuó en esos 57 casos fueron la mononucleosis (14 casos, ó 24,6\%), la hepatitis (del tipo A, B o C; cuatro casos, ó 7,0\%) y la infec- 
CUADRO 4. Análisis clínicos de laboratorio de 57 pacientes hospitalizados con dengue hemorrágico confirmado por laboratorio, Puerto Rico, 1990-1991

\begin{tabular}{|c|c|c|c|c|}
\hline \multirow[b]{2}{*}{$\begin{array}{l}\text { Pruebas con } \\
\text { resultados anormales }\end{array}$} & \multicolumn{2}{|c|}{ Pacientes } & \multicolumn{2}{|c|}{ Resultados } \\
\hline & $\begin{array}{l}\text { Positivos/ } \\
\text { Investigados }\end{array}$ & (\% positivo) & Promedio ${ }^{a}$ & Recorrido \\
\hline $\begin{array}{l}\text { Trombocitopenia } \\
\text { Recuento de plaquetas } \\
\leq 100,000 / \mathrm{mm}^{3}\end{array}$ & $57 / 57$ & $(100,0)$ & 45980 & $(9$ a 99000$)$ \\
\hline $\begin{array}{l}\text { Permeabilidad capilar } \\
\text { aumentada } \\
\text { Hemoconcentración } \geq 0,20 \\
\text { Hiposeroproteinemia } \\
\text { Hiposeroalbuminemia }\end{array}$ & $\begin{array}{l}34 / 57 \\
18 / 51 \\
35 / 52\end{array}$ & $\begin{array}{l}(59,6) \\
(35,3) \\
(67,3)\end{array}$ & $\begin{array}{l}0,26 \\
6,3 \\
3,5\end{array}$ & $\begin{array}{c}(0 \text { a } 1,0) \\
(3,8 \text { a } 8,3) \\
(2,3 \text { a } 4,9)\end{array}$ \\
\hline $\begin{array}{l}\text { Compromiso hepático } \\
\text { Aumento de ASAT (SGOT) } \\
\text { Aumento de ALAT (SGPT) } \\
\text { Bilirrubina total } \\
>1 \mathrm{mg} / \mathrm{dL}\end{array}$ & $\begin{array}{l}42 / 50 \\
34 / 48\end{array}$ & $\begin{array}{l}(84,0) \\
(70,8) \\
(11,1)\end{array}$ & $\begin{array}{l}260 \\
242\end{array}$ & $\begin{array}{r}(19 \text { a } 13 \text { 368) } \\
(118 \text { a } 2607) \\
(0,1 \text { a } 21,2)\end{array}$ \\
\hline $\begin{array}{l}\text { Sangrado }{ }^{\mathrm{b}} \\
\text { TP }>25 \% \text { del control } \\
\text { TPT }>25 \% \text { del control }\end{array}$ & $\begin{array}{r}2 / 43 \\
18 / 43\end{array}$ & $\begin{array}{r}(4,7) \\
(41,9)\end{array}$ & $\begin{array}{l}13 \% \text { más que el control } \\
33 \% \text { más que el control }\end{array}$ & $\begin{array}{c}(-10 \text { a } 390 \%) \\
(0 \text { a } 249 \%)\end{array}$ \\
\hline
\end{tabular}

${ }^{a}$ Resultado promedio en los casos que se sometieron a pruebas.

${ }^{b}$ ASAT - Aspartato aminotransferasa, llamada anteriormente transaminasa glutamicooxalacética (SGOT); ALAT - Alanino aminotransferasa, llamada anteriormente transaminasa glutamicopirúvica (SGPT); TP - tiempo de protrombina; TPT - tiempo parcial de tromboplastina.

ción por citomegalovirus (tres casos, ó $5,3 \%$ ). Ninguna de las respectivas pruebas de laboratorio arrojó resultados positivos. Otros estudios diagnósticos realizados en esos pacientes correspondieron a aspiración de médula ósea (cinco pacientes, ó 8,8\%), exploración con tomografía computadorizada (cuatro pacientes, ó 7,0\%) y ultrasonografía (cuatro pacientes, ó $7,0 \%$ ). Para todos los pacientes con $\mathrm{DH}$, el tratamiento incluyó la sustitución de líquidos por vía endovenosa y, frecuentemente, también los esteroides ( 16 casos, ó $28,1 \%$ ) y las transfusiones de plaquetas (13 casos, ó 22,8\%). Dos pacientes $(3,5 \%)$ recibieron inmunoglobulina endovenosa y dos $(3,5 \%)$ recibieron plasma fresco congelado.

\section{DISCUSIÓN}

Esta investigación constituye la primera descripción de las características clínicas de los casos de $\mathrm{DH}$ endémico en Puerto Rico que se identificaron mediante la revisión de los registros hospitalarios de los casos notificados por los sistemas paralelos de vigilancia del dengue en laboratorios y hospitales de la isla. La observada incidencia de $\mathrm{DH}$ con diagnóstico positivo según pruebas de laboratorio fue casi tres veces superior a la detectada por el sistema de vigilancia (57 y 20 casos, respectivamente); más aún, la cifra mayor parece haber representado solo una mínima fracción de todos los casos que ocurrieron. De los casos de DH positivos según pruebas de laboratorio, $59,6 \%$ egresaron sin diagnóstico de DH; una defunción había sido atribuida no a dengue, sino a insuficiencia hepática y hepatitis. Además, 29 casos con un posible diagnóstico de DH no remitieron muestras apropiadas para diagnosticar la enfermedad. Esto evidentemente indica que es necesario mejorar la capacidad de los médicos de reconocer el DH.

De los 115 pacientes cuyos registros clínicos fueron compatibles con un diagnóstico de FD, 53 tuvieron resultados positivos en las pruebas de laboratorio. En 15 (28,3\%) de los 53 se emitió un diagnóstico de $\mathrm{DH}$ al egreso; cabe señalar que en los registros de 11 de ellos no constaban signos de excesiva permeabilidad capilar. Eso indica que, por lo común, la permeabilidad capilar no es un criterio tenido en cuenta para el diagnóstico de DH. Hubo, además, seis defunciones entre los 115 pacientes, tres de ellas en los 53 con resultados de laboratorio positivos. Esa alta tasa de letalidad (3/53 ó 5,7\%) en pacientes de $\mathrm{DH}$ sugiere que en dicho grupo no se incluyeron casos de $\mathrm{DH}$ que no llegaban a satisfacer los criterios de definición, bien sea por falta de información o porque (como se notificó en otros lugares) las infecciones por dengue de evolución mortal a veces cursan sin escape de líquidos por las paredes de los capilares (11). Sea cual fuere el caso, se impone un diagnóstico temprano y preciso de $\mathrm{DH}$, no solo con fines de vigilancia sino también para prevenir el choque y la muerte consecutiva.

En Puerto Rico, las características clínicas de los casos de DH con positividad en las pruebas de laboratorio, así como el perfil de su progresión, son similares a los de los casos notificados en países con graves epidemias o endemia prolongada. Otras descripciones han señalado a la trombocitopenia como signo premonitorio de escape capilar, hemoconcentración e hipotensión. Nuestros datos (que se basan en el tiempo transcurrido hasta la detección de los valores extremos, y no hasta el primer valor anormal) son compatibles con este punto (37-42).

En contraste con lo relatado por otros informes, la hematuria microscópica fue la manifestación hemorrágica detectada con más frecuencia $(51,9 \%$ de los 57 casos de DH), en tanto que la prueba del torniquete fue positiva en solo $5(31,3 \%)$ de los 16 pacientes en quienes se practicó. Ambos resultados reflejan las prácticas clínicas en Puerto Rico, donde el amplio acceso a pruebas químicas y microscópicas automatizadas para la detección de hematuria explica el uso poco frecuente de la prueba del torniquete. La hematuria fue también prominente en la epidemia ocurrida en Tahití en 1971 y en los 29 casos de DH notificados durante la epidemia que afectó a Puerto Rico en 1986 y $1987(43,44)$. 
La hepatomegalia ha sido comúnmente referida en niños con $\mathrm{DH}$ en Indonesia, India y Tailandia (64,9 a $90,0 \%)(37,45,46)$, pero solo se observó en seis $(10,5 \%)$ de los 57 casos de $\mathrm{DH}$ aquí notificados. Al igual que en nuestro estudio, la hepatomegalia fue observada en una minoría de los casos de $\mathrm{DH}$ estudiados en Indonesia en 1976, en Cuba en 1981, en Manila en 1983-1984 y en la Isla de Hainán (China) en 1985 (5,2 a 19,4\%) (38-40, 47).

La elevación de las enzimas hepáticas observada en pacientes de Puerto Rico fue muy similar a la descrita en pacientes con FD en Taiwán en 1987-1988, así como en los hospitalizados con diagnóstico clínico de dengue en Puerto Rico en 1991 (48, 49). En otros informes recientes se ha destacado la relación entre la infección por dengue, trastornos hepáticos y encefalopatía (50-52). Los tres pacientes con SCD o DH grado IV (de los cuales dos fallecieron) tuvieron las mayores concentraciones de ASAT encontradas en los 57 casos de DH aquí descritos.

Como era de esperar, todos los 57 pacientes fueron tratados con líquidos por vía endovenosa y solo dos $(3,5 \%)$ recibieron plasma. La administración de plaquetas, esteroides e inmunoglobulina endovenosa fue motivada por una trombocitopenia grave. Por lo común, el estado del paciente exige que el médico tome una decisión sobre el tratamiento antes de recibir los resultados de las pruebas de laboratorio detectoras de dengue $\mathrm{u}$ otros procesos patológicos (53).

Como lo indican otros análisis del papel que desempeña la respuesta inmune en la gravedad de los síntomas, pudimos documentar que en la mayoría de los casos confirmados, la respuesta inmune del paciente al virus indicaba la presencia de infección secundaria (54). La distribución del DH por edad en Puerto Rico y en otros países de las Américas parece ser muy distinta a la observada en el Asia Sudoriental, donde la población ha estado expuesta al dengue endémico y a epidemias frecuentes por varios decenios $(41,42,55,56)$. En Indonesia, por ejemplo, la mediana de edad de los pacien- tes que tuvieron DH de 1979 a 1984 fue de 4 años y 11 meses (57). En cambio, en Puerto Rico la mediana de edad fue de 38 años y tuvo un recorrido de 4 a 86. Aunque en otros años se han documentado defunciones por dengue en niños de Puerto Rico, no ocurrió ninguna defunción atribuible a esta enfermedad en los 2 años que duró el presente estudio (44).

Casi todas las descripciones de casos de $\mathrm{DH}$ provienen de pacientes evaluados en un hospital o de centros para la investigación del dengue situados en zonas donde la incidencia de la enfermedad es alta. En cambio, la presente descripción se basa en una combinación de sistemas de vigilancia pasiva y activa establecidos para orientar las actividades de control de la enfermedad en Puerto Rico (20). Por lo general, el diagnóstico de dengue depende de la recolección repetida y oportuna $\mathrm{y}$ del examen inmediato de las muestras de suero. Estas condiciones rara vez se dan en la atención clínica ordinaria y es imposible cumplirlas en casos en que el paciente muere después de una breve enfermedad.

Como lo indica la comparación de la media de los intervalos de tiempo transcurridos desde el comienzo de la enfermedad hasta el egreso del hospital en los tres grupos de pacientes que hemos estudiado, la brevedad del período disponible para el examen clínico es un grave obstáculo para el diagnóstico y la notificación del dengue. Esto contribuye a explicar la baja proporción de casos confirmados por laboratorio $(15 / 57=26 \%)$ en pacientes de $\mathrm{DH}$ en quienes se llevó a cabo un diagnóstico de laboratorio, así como la elevada proporción de casos indeterminados $(29 / 102=28 \%)$ que habían recibido un diagnóstico clínico de $\mathrm{DH}$. La notificación inexacta de la fecha en que comenzaron los síntomas puede llevar también a un diagnóstico equivocado, ya que los casos serán erróneamente clasificados como negativos por laboratorio en lugar de indeterminados, si el intervalo entre el comienzo real de la enfermedad y la recolección de las muestras de sangre ha sido demasiado corto para detectar un aumento de magnitud diagnóstica del título de anticuerpos.

La causa de las enfermedades que se ciñeron a los criterios clínicos de $\mathrm{DH}$ pero que no arrojaron resultados positivos por laboratorio no han podido definirse sobre la base de los datos disponibles para este estudio. Sin embargo, parece razonable atribuir al dengue una gran proporción de los casos indeterminados, en tanto que los restantes, junto con los negativos, podrían haberse debido a enfermedades infecciosas con síntomas similares a los del dengue (e.g., leptospirosis, sarampión, influenza y muchas otras), o bien a la coincidencia de un episodio febril agudo con una enfermedad no infecciosa (e.g., leucemia).

En síntesis, las características clínicas de los casos de DH positivos por laboratorio que ocurrieron en Puerto Rico entre 1990-1991 fueron similares a las de los casos descritos en otros países. No obstante, la distribución por edad de esos casos siguió el patrón observado en las Américas, donde todos los grupos de edad son afectados, en contraposición al observado en el Asia Sudoriental, donde la enfermedad afecta sobre todo a los niños pequeños. El número de casos de $\mathrm{DH}$ identificados por medio de este estudio fue 2,85 veces mayor que el notificado, y es posible que el número real de casos ocurridos haya sido aun mayor. En consecuencia, se hace evidente que tanto el reconocimiento como la notificación de $\mathrm{DH}$ a cargo de los médicos clínicos deben mejorarse.

Agradecimiento. Los autores desean destacar el sentido de anticipación de Edward B. Hayes y Duane J. Gubler, quienes establecieron el sistema hospitalario de vigilancia del dengue grave en que se basó este estudio. Además, expresan su reconocimiento al personal del laboratorio de la Sección de Dengue, Centros para el Control y la Prevención de Enfermedades de los Estados Unidos, por su colaboración, que fue decisiva para la identificación de los casos incluidos en el presente estudio. 


\section{REFERENCIAS}

1. Institute of Medicine. Emerging infections: microbial threats to health in the United States. Washington, DC: National Academy Press; 1992.

2. Centers for Disease Control and Prevention. Addressing emerging infectious disease threats: a prevention strategy for the United States. Atlanta: U.S. Department of Health and Human Services, Public Health Service; 1994.

3. Halstead SB. The XXth century dengue pandemic: need for surveillance and research. World Health Stat Q 1992;45:292-298.

4. Nimmannitya S. Clinical spectrum and management of dengue haemorrhagic fever. Southeast Asian J Trop Med Public Health 1987; 18:392-397.

5. Sumarmo, Wuryadi S, Gubler DJ. Clinical observations on hospitalized patients with virologically confirmed dengue hemorrhagic fever in Jakarta, Indonesia 1975-1983. Paediatr Indones 1986;26:137-151.

6. Chin CK. Outpatient management of dengue infection in the University Hospital, Kuala Lumpur. Malays J Pathol 1993;15:21-23.

7. Rohde JE. Clinical management of severe dengue. Trop Doct 1978;8:54-61.

8. Tassniyom S, Vasanawathana V, Chirawatkul A, Rojanasuphot S. Failure of high-dose methylprednisolone in established dengue shock syndrome: a placebo-controlled, doubleblind study. Pediatrics 1993;92:111-115.

9. Lum LCS. Management of dengue haemorrhagic fever/dengue shock syndrome. Malays J Pathol 1993;15:29-33.

10. Yong BH. Critical care aspects of dengue haemorrhagic fever/dengue shock syndrome. Malays J Pathol 1993;15:35-39.

11. Sumarmo, Wulur H, Jahja E, Gubler DJ, Suharyono W, Sorensen K. Clinical observations on virologically confirmed fatal dengue infections in Jakarta, Indonesia. Bull World Health Organ 1983;61:693-701.

12. López Correa RH, Cline BL, Ramírez-Ronda C, Bermúdez R, Sather GE, Kuno G. Dengue fever with hemorrhagic manifestations: a report of three cases from Puerto Rico. Am J Trop Med Hyg 1978;27:1216-1224.

13. Woodall JP, López-Correa RH, Sather GE, Moore CG. The absence of epidemic dengue hemorrhagic fever from the Americas. En: Hotta S, ed. Dengue hemorrhagic fever 1981: proceedings of the First International Center for Medical Research Seminar 1980, Nov 21-22, Kobe, Japan. Kobe: ICMR; 95-106.

14. Álvarez $\mathrm{ME}$, Ramírez-Ronda $\mathrm{CH}$. Dengue and hepatic failure. Am J Med 1985;79:670-674.

15. Gubler DJ. Dengue and dengue hemorrhagic fever in the Americas. P R Health Sci J 1987;6: 107-111.

16. Ramírez-Ronda CH. Dengue in Puerto Rico: clinical manifestations and management from the 1960s to 1987. P R Health Sci J 1987;6: 113-118.

17. Pan American Health Organization. Dengue in the Americas: an update. Epidemiol Bull (PAHO) 1993;14:1-3.

18. Rigau-Pérez JG, Gubler DJ, Vorndam AV, Clark GG. Dengue surveillance: United States, 1986-1992. En: Centers for Disease Control and Prevention. CDC surveillance summaries. 22 July 1994;43(No. SS-2):7-19.

19. World Health Organization. Dengue haemorrhagic fever: diagnosis, treatment, and control. Geneva: WHO; 1986:12-14

20. Gubler DJ. Surveillance for dengue and dengue hemorrhagic fever. Bull Pan Am Health Organ 1989;23:397-404.

21. Gubler DJ, Casta-Vélez A. A program for prevention and control of epidemic dengue and dengue hemorrhagic fever in Puerto Rico and the U.S. Virgin Islands. Bull Pan Am Health Organ 1991;25:237-247.

22. Jordan CD, Flood JG, Laposata $M$, Lewandrowski KB. Normal reference laboratory values. N Engl J Med 1993;327:718-724.

23. Anónimo. JAMA instructions for authors. JAMA 1995;273:27-34.

24. Yager HM, Harrington JT. Urinalysis and urinary electrolytes. En: Jacobson HR, Striker GE, Klahr S, eds. The principles and practice of nephrology. Philadelphia: Decker; 1991:171.

25. Rosen L, Gubler DJ. The use of mosquitoes to detect and propagate dengue viruses. Am J Trop Med Hyg 1974;23:1153-1160.

26. Gubler DJ, Kuno G, Sather GE, Vélez M Oliver A. Mosquito cell cultures and specific monoclonal antibodies in surveillance for dengue viruses. Am J Trop Med Hyg 1984;33: $158-165$

27. Kuno G, Gubler DI, Vélez M, Oliver A. Comparative sensitivity of three mosquito cell lines for isolation of dengue viruses. Bull World Health Organ 1985;63:279-286.

28. Burke DS, Nisalak A, Ussery MA. Antibody capture immunoassay detection of Japanese encephalitis virus immunoglobulin $\mathrm{M}$ and $\mathrm{G}$ antibodies in cerebrospinal fluid. J Clin Microbiol 1982;15:1034-1042.

29. Gubler DJ, Sather GE. Laboratory diagnosis of dengue and dengue hemorrhagic fever. En: Fonseca da Cunha F, ed. Simposio Internacional sobre Febre Amarela e Dengue, 1988. Rio de Janeiro: Fundação Oswaldo Cruz/BioManguinhos; 1990:291-322.

30. Kuno G, Gómez I, Gubler DJ. Detecting artificial anti-dengue $\operatorname{IgM}$ immune complexes using an enzyme-linked immunosorbent assay. Am J Trop Med Hyg 1987;36:153-159.

31. Chungue E, Marché G, Plichart R, Boutin JP, Roux J. Comparison of immunoglobulin $G$ enzyme-linked immunosorbent assay (IgGELISA) and haemagglutination inhibition (HI) test for the detection of dengue antibodies: prevalence of dengue IgG-ELISA antibodies in Tahiti. Trans $R$ Soc Trop Med Hyg 1989; 83:708-711.

32. Clarke DH, Casals J. Techniques for hemagglutination and hemagglutination-inhibition with arthropod-borne viruses. Am I Trop Med Hyg 1958;7:561-573.

33. Hall WC, Crowell TP, Watts DM, Barros VL, Kruger H, Pinheiro F, et al. Demonstration of yellow fever and dengue antigens in formalin-fixed paraffin-embedded human liver by immunohistochemical analysis. Am J Trop Med Hyg 1991:45:408-417.

34. Gubler DJ, Suharyono W, Lubis I, Eram S, Sulianti Saroso J. Epidemic dengue haemor- rhagic fever in rural Indonesia: I. virological and epidemiological studies. Am J Trop Med Hyg 1979;28:701-710.

35. Centers for Disease Control. Case definitions for public health surveillance. MMWR 1990; 39:RR-13, 10-11.

36. Lewis JG, Chang G-J, Lanciotti RS, Kinney RK, Mayer LW, Trent DW. Phylogenetic relationships of dengue-2 viruses. Virology 1993; 197:216-224.

37. Nimmannitya S, Halstead SB, Cohen SN, Margiotta MR. Dengue and chikungunya virus infection in man in Thailand, 1962-64: I. observations on hospitalized patients with hemorrhagic fever. Am J Trop Med Hyg 1969; 18:954-971.

38. Hayes CG, Manaloto CR, Gonzales A, Ranoa $\mathrm{CP}$. Dengue infections in the Philippines: clinical and virological findings in 517 hospitalized patients. Am J Trop Med Hyg 1988;39: 110-116.

39. Qiu F-X, Gubler DJ, Liu J-C, Chen Q-Q. Dengue in China: a clinical review. Bull World Health Organ 1993;71:349-359.

40. Guzmán MG, Kourí GP, Bravo J, et al. Dengue haemorrhagic fever in Cuba: II. clinical investigations. Trans R Soc Trop Med Hyg 1984;78: 239-241.

41. Rosso F, Restrepo de Meza MT, Alzate A Muñoz J, Moreno $\mathrm{CH}$. Dengue hemorrágico en el Hospital Universitario del Valle, 1990-1992. Colombia Med 1994;25:10-14.

42. Zagne SMO, Alves VGF, Nogueira RMR Miagostovich MP, Lampe E, Tavares W. Dengue haemorrhagic fever in the state of Rio de Janeiro, Brazil: a study of 56 confirmed cases. Trans R Soc Trop Med Hyg 1994;88: 677-679.

43. Moreau J-P, Rosen L, Saugrain J, Lagraulet J. An epidemic of dengue on Tahiti associated with hemorrhagic manifestations. Am J Trop Med Hyg 1973;22:237-241.

44. Dietz VJ, Gubler DJ, Ortiz S, et al. The 1986 dengue and dengue hemorrhagic fever out break in Puerto Rico: epidemiologic and clinical observations. P R Health Sci J 1996;15: 201-210.

45. Munir M, Husada T, Mustadjab I. Dengue haemorrhagic fever: a problem of clinical diagnosis and proposal for using a scoring system. Paediatr Indones 1982;22:11-22.

46. Srivastava VK, Suri S, Bhasin A, Srivastava L, Bharadwaj M. An epidemic of dengue haemorrhagic fever and dengue shock syndrome in Delhi: a clinical study. Ann Trop Paediatr 1990; 10:329-334

47. Eram S, Setyabudi Y, Sadono TI, Sutrisno DS, Gubler DJ, Sulianti Saroso J. Epidemic dengue hemorrhagic fever in rural Indonesia: II. clinical studies. Am I Trop Med Hyg 1979;28: 711-716.

48. Kuo CH, Tai DI, Chang-Chien CS, Lan CK, Chiou SS, Liaw YF. Liver biochemical tests and dengue fever. Am J Trop Med Hyg 1992;47: 265-270.

49. García S, Morales R, Hunter RF. Dengue fever with thrombocytopenia: studies towards defining vulnerability of bleeding. Bol Asoc Med P R 1995;87:2-7. 
50. Innis BL, Myint KSA, Nisalak A, Ishak KG, Nimmannitya S, Laohapand T, et al. Acute liver failure is one important cause of fatal dengue infection. Southeast Asian I Trop Med Public Health 1990;21:695-696.

51. Lum LCS, Lam SK, George R, Devi S. Fulminant hepatitis in dengue infection. Southeast Asian J Trop Med Public Health 1993;24:467-471.

52. Rajajee S, Mukundan D. Neurological manifestations in dengue hemorrhagic fever. Indian Pediatr 1994;31:688-690.

53. College of American Pathologists. Practice parameter for the use of fresh-frozen plasma, cryoprecipitate, and platelets. JAMA 1994;271: 777-781.

54. Halstead SB. Pathogenesis of dengue: challenges to molecular biology. Science 1988;239: 476-481.

55. Kourí GP, Guzmán MG, Bravo JR, Triana C. Dengue haemorrhagic fever/dengue shock syndrome: lessons from the Cuban epidemic, 1981. Bull World Health Organ 1989;87: 375-380.

56. Communicable Diseases Program, PAHO. Dengue hemorrhagic fever in Venezuela. Epidemiol Bull (PAHO) 1990;11(2):7-9.
57. Nathin MA, Harun SR, Sumarmo. Dengue haemorrhagic fever and Japanese B encephalitis in Indonesia. Southeast Asian J Trop Med Public Health 1988;19:475-481.

Manuscrito recibido el 10 de abril de 1996 y aceptado para publicación en versión revisada el 21 de agosto de 1996.

ABSTRACT The aim of the study reported here was to demonstrate that dengue hemorrhagic fever (DHF) occurs in Puerto Rico, that it is underreported, and that this underreporting is due partly to underdiagnosis in hospitals. Surveillance for severe dengue identified 986 hospitalizations for suspected dengue in 1990-1991. At the time, the surveillance system routinely identified $20 \mathrm{DHF}$ cases, including three with dengue shock syndrome (DSS). Our subsequent review of these 986 patients' hospital records identified 102 whose records supported a clinical diagnosis of DHF (88) or DSS (14). Of these 102, 57 had positive virologic or serologic results for dengue and met the World Health Organization criteria for DHF (fever, hemorrhagic manifestations, thrombocytopenia, and excessive capillary permeability). This group of 57 patients had a mean age of 38 years, contained a preponderance of males $(34,59.3 \%)$, included eight cases of DSS, and involved two (3.5\%) fatalities (in females 16 and 55 years old). Hemorrhagic manifestations were mild; hemoconcentration, hypoalbuminemia, and elevated aspartate and alanine aminotransferase levels were frequently encountered. The median duration of hospitalization was five days. The clinical description of these laboratory-positive DHF cases in Puerto Rico is consistent with previous descriptions of DHF in the medical literature; but the patients' age distribution is similar to the pattern typically found in the Americas (where all age groups tend to be affected), as opposed to Southeast Asia (where mostly small children are affected). The number of DHF cases identified by our study was nearly three times that reported through the established surveillance system. Our findings indicate that recognition and reporting of DHF by local clinicians needs to be improved. 